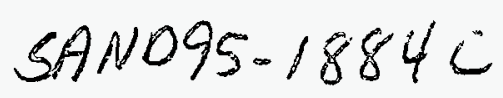

\title{
ANALYSIS OF A HIGH INTENSITY X-RAY SOURCE USING A SPECIALIZED DOPPLER INTERFEROMETER SYSTEM
}

\author{
K. J. Fleming \\ Explosive Projects \& Diagnostics, Dept 2554, Sandia National Laboratories, Albuquerque, NM, USA 87123 \\ The Saturn accelerator at Sandia National Laboratories is a high power, variable-spectrum, $x$-ray source \\ capable of simulating radiation effects of nuclear countermeasures on electronic and material components of \\ space systems. It can also function as a pulsed-power and radiation source, and as a diagnostic test bed for \\ a variety of applications. Obtaining highly accurate measurements of the emission spectra is difficult \\ because the high intensity $\mathrm{X}$-rays and MegaAmpere levels of current inside the experiment chamber can \\ damage or destroy electronic measurement devices. For these reasons, an optical based measurement \\ system has been designed, developed and successfully tested in the Saturn accelerator. The sysiem uses \\ fiber optic coupled sensor(s) connected to a specialized Doppler interferometer system which analyzes the \\ shock wave imparted into a target material. This paper describes the optical system, its related components, \\ and material response data of polymethyl methacrylate.
}

\section{INTRODUCTION}

$\mathrm{X}$-ray emissions produced by the Saturn accelerator have an important role in radiation effects simulation, especially since the future of underground nuclear testing is uncertain. Accurate modeling and testing must rely on precise measurements of the response of materials subjected to the $\mathrm{x}$-ray radiation. For this reason, a "fidelity measurement series" is underway. This series of experiments uses different types of devices that measure material response to intense. short pulse, $x$ ray radiation. All of these measurement devices are simultaneously exposed to the same radiation source.

This test series uses the Saturn accelerator in the Plasma Radiation Source configuration which has an argon line energy of $3.2 \mathrm{keV}$. This scheme ascertains the most accurate measurements possible since other (different) devices such as quartz and PVDF gauges are simultaneously measuring emission output. The VISAR (Velocity Interferometer System for Any Reflector) is the only system in this series which uses optical methods for analyzing material response to $x$-ray emissions. An optically transparent material with known shock properties is exposed to the $x$-ray source. The energy is absorbed by the material causing a shock wave which is analyzed. This paper will describe the method of particle velocity measurement, the sensor design, the complete system configuration, and the data obtained.

\section{BACKGROUND}

The VISAR is a unique measurement tool in that its measurement method is based on optical, not electronic phenomena, which gives it immunity from electrical interference. The bandwidth of the system is only limited by the detectors and recording instrumentation. Because VISAR uses laser light for measurement, it is non-intrusive. The VISAR was developed by Barker and Hollenbach to perform shock wave studies on various materials. In its original configuration, the test material was impacted with a projectile and the resulting shock wave was analyzed by the VISAR (I). In the Saturn experiment, similar measurement techniques are used but the shock wave energy of the source is replaced by the intense $x$-ray radiation. 


\section{DISCLAIMER}

Portions of this document may be illegible in electronic image products. Images are produced from the best available original document. 


\section{THEORY OF OPERATION}

The primary components of VISAR are a single frequency laser, a modified Michelson interferometer cavity, high speed photomultiplier tubes and a recording device such as a digitizing oscilloscope. The laser used for this experiment is an argon-ion with a 1 Watt continuous wave (cw) output at 514 $\mathrm{nm}$ wavelength. The material exposed to the $\mathrm{x}$-rays is polymethyl methacrylate (PMMA), specified as Polycast UVB with a density of $1.184 \mathrm{~g} / \mathrm{cm}^{3}$. A 50 $\mathrm{mm}$ diameter, $1 \mathrm{~mm}$ thick PMMA disk is cemented to a 50-mm diameter, $12.5 \mathrm{~mm}$ thick PMMA disk with a thin $(25 \mu \mathrm{m})$ aluminum foil sandwiched between the two disks. The foil and disks are bonded together under pressure with a very thin layer of epoxy glue (This assembly is referred to as $\mathrm{a}^{-}$ "target"). A special fiber optic coupled sensor (2) is attached to the target assembly such that the laser light is focused through the $12.5 \mathrm{~mm}$ thick PMMA disk onto a point on the foil (Fig. 1). The diffuse surface of the foil reflects the light which is collected by the sensor and focused into another fiber optic which is connected to the VISAR interferometer cavity.

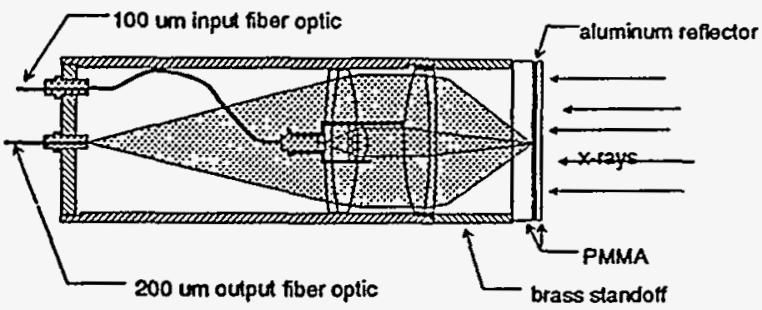

FIGURE 1. Sensor configuration. for $x$-ray analysis. Source is approximately $100 \mathrm{~mm}$ from target sample.

The VISAR cavity is a modified, unequal leg Michelson interferometer. The sensitive components in the cavity are cemented together to significantly reduce the need for alignment (Fig. 2). The light from the output fiber optic, connected to the sensor, is collimated and sent through the cavity. The beamsplitting coating reflects one beam through the glass "delay bar" and the other beam through air and a 1/8 wave retarder. The beams are recombined to form two sets of interference patterns which have simultancous intensities 180 degrees out of phase with each other. The interfered light is routed to optical detectors which convert optical signals to electric signals that are then transmitted to the recording device(s). The inverted-phase light is electronically inverted and added to the normal phase light which doubles the signal intensity and cancels out most of the self light generated in the irradiated fiber optics (3). A 20 angstrom bandpass filter placed in front of the return fiber optic further filters the laser light to keep the signal-to-noise ratio high.

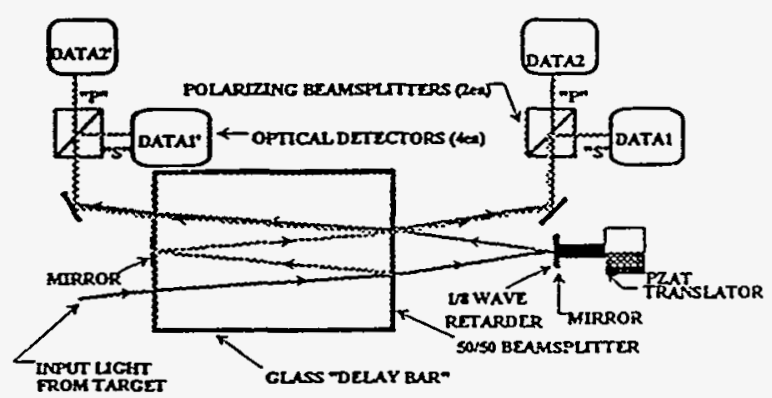

Figure 2. Drawing of "Fixed Cavity" interferometer. "DATA X" are optical detectors. The PZAT Translator simulates the return signal and fine adjusts the interferometer.

When the $x$-ray radiation exposes the PMMA, it generates a shock wave near the front surface that travels into the sample. This compressive wave propagates through the aluminum foil and into the rear PMMA disk. The resulting instantaneous particle velocity (PV) at the foil/PMMA interface causes a Doppler shift in the light: Since the length of the legs of the interferometer cavity are different, the Doppler shift arrives at the beam recombination point at different times. This relationship is defined by:

arrival time $=h(1-1 / n)$

where $h$ is the delay leg length and $n$ is the index of refraction. The distance the light travels in the delay leg is longer than in the reference leg and the speed of light is slower in glass than in air. Using the relationship from equation (l), the delay time $\tau$ is :

$$
\tau=2 h / c=\frac{2 h(1-1 / n)}{c}
$$


where $c$ is the speed of light in a vacuum. Using these relationships, the fringe count $F(t)$ relates to target velocity $u(t-\tau / 2)$ as:

$u(t-t / 2)=\frac{\lambda F(t)}{2 \tau(1+\Delta v / v)} \cdot \frac{1}{1+\delta}$

in which $\lambda$ is the wavelength of the laser light, $\Delta v / v$ is an index of refraction correction factor for the window, $\delta$ is a correction factor with respect to wavelength for dispersion in the delay bar. Equation (3) is manipulated to obtain the velocity-per-fringe (VPF) constant for an interferometer which is:

$V P F=\frac{\lambda}{2 \tau(1+\Delta v / v)} \cdot \frac{1}{1+\delta}$

\section{EXPERIMENT DESCRIPTION}

The VISAR for the Saturn experiments is a dualleg system which consists of two interferometer cavities with different VPFs. Leg 1 has a VPF of 38 $\mathrm{m} / \mathrm{s}$, and Leg 2 has a VPF of $108 \mathrm{~m} / \mathrm{s}$. The laser light collected from the sample is split and routed to the two interferometer cavities (Fig.3). This dual leg technique gives a broader measurement range for unknown velocities, and can catch very fast shock jumps that may be missed with a single leg system.

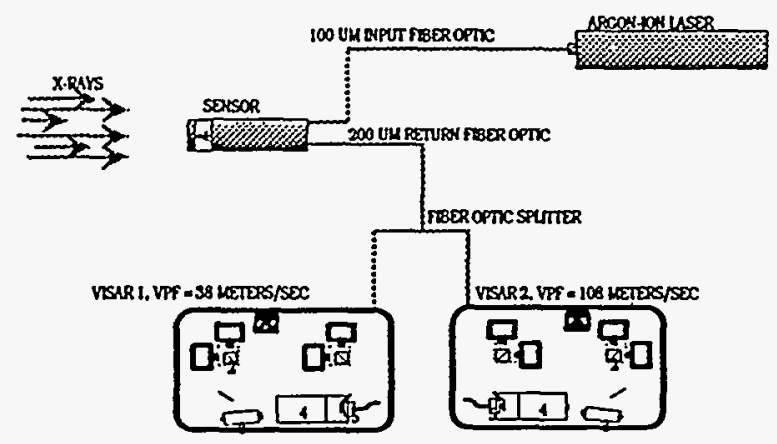

Figure 3. Diagram of Saturn VISAR experiment using the dual interferometer cavity configuration. The sensor assembly is $50 \mathrm{~mm}$ diameter, $200 \mathrm{~mm}$ long. The fiber optic length is 100 meters.

The digitizing oscilloscopes have a 2 Gigasample rate. A total of 32,000 points per trace were recorded, although most of the useful data are contained in about 5000 points. The data reduction software converts the sinusoidal traces to a polar plot, then to a velocity as a function of time plot.

\section{DATA REDUCTION}

The Hugoniot of PMMA is the relationship between the shock and particle velocities of the material and is expressed as :

$U_{s}=C_{o}+S U_{p}$

where:

$\mathrm{U}_{\mathrm{s}}$ is the shock velocity in $\mathrm{mm} / \mu \mathrm{s}$

$\mathrm{C}_{\mathrm{o}}$ is the initial bulk sound velocity in $\mathrm{mm} / \mu \mathrm{s}$

$S$ is a dimensionless empirical parameter

$U_{p}$ is the particle velocity in $\mathrm{mm} / \mu \mathrm{s}$

For PMMA, equation 5 has the form (4):

$U_{s}=2.49+1.69 U_{p}$

\section{RESULTS}

Figure 4 on the next page shows the particle velocity data from one of the Saturn shots. Both VISAR legs have been simultaneously plotted to show any measurement differences in the velocity history. In this case, the peak velocity is 86 metersper-second with a measurement tolerance of $4 \%$. The high bandwidth of VISAR is valuable in tracking the fast $(\approx 10 \mathrm{~ns})$ compression and release components of the shock wave. Both VISAR legs tracked the entire shock history with little differences in the measured particle velocities.

The measured particle velocity can be plotted as a function of shock velocity. By knowing the values of shock velocity, particle velocity, and density, the pressure can be calculated using conservation of momentum:

$\mathrm{P}-\mathrm{P}{ }_{\mathrm{o}}=U_{s} U_{p} \rho_{o}$

where:

$\mathrm{P}$ is the final pressure in $\mathrm{GPa}$

$P_{0}$ is the initial pressure, which is equal to zero.

$\rho_{o}$ is the initial density in $\mathrm{g} / \mathrm{cc}$ (in this case $=1.85$ ). 
During shock loading, the stress at any time in the event can now be calculated. For this example, the peak pressure is $.314 \mathrm{GPa}$. It is important to note that equation 7 should not be used in unloading because the relationship between $U_{s}$ and $U_{p}$ differs from that of equation 6 and is not currently documented.

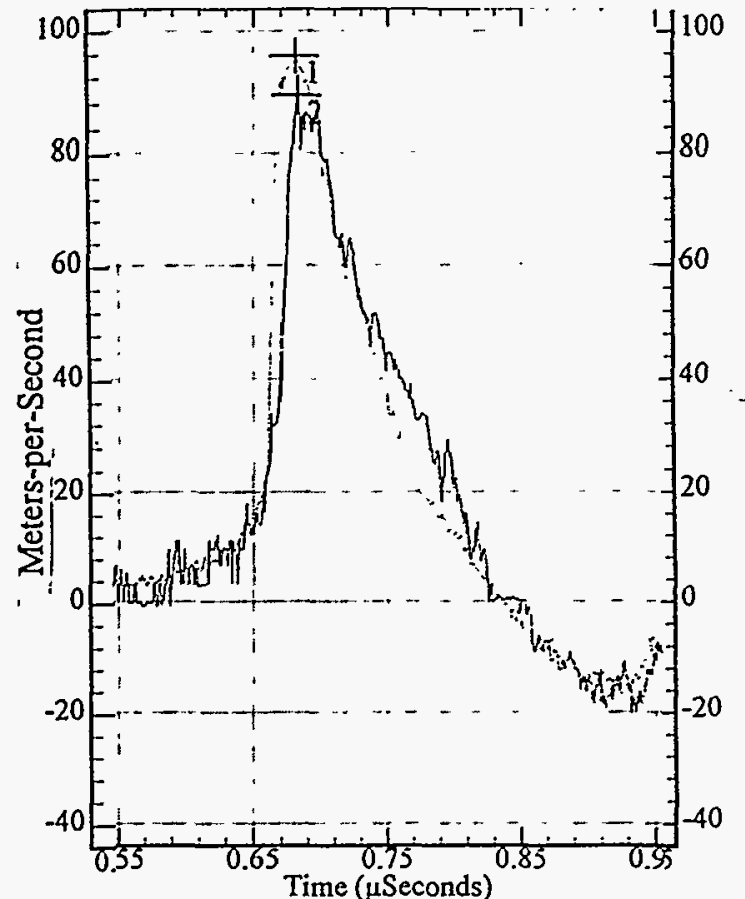

Figure 4. Particle velocity in the PMMA sample. Peak pressure is calculated to be $.314 \mathrm{GPa}$.

\section{CONCLUSION}

The VISAR coupled sensors worked well, performing with the predicted immunity from electrical interference. The radiation induced selflight was blocked out by using the inherent light filtering nature of VISAR and narrow optical bandpass filters. Radiation induced fiber darkening was only detrimental after three or more exposures to $x$-ray radiation, so an efficient method of fiber optic replacement was developed.

The unique measurement technology of VISAR has brought material response analysis to a new level, since particle velocity can be measured with a high level of detail and accuracy. The measurements are repeatable with results closely matching predicted pressures in PMMA and measurements using piezoelectric gauges. This technology is in the early stages of development and more tests using different materials are planned for the future.

\section{ACKNOWLEDGMENTS}

This work was performed at Sandia National Laboratories for the US Department of Energy under contract DE-AC04-94Alb5000.

Developing new diagnostic techniques in a limited time frame is a very challenging goal requiring hard work and highly competent support personnel. The author gratefully thanks the following people for sharing their talent and hard work to make this a successful venture.

Ed Vieth-Fiber optic fabrication, diagnostics. Richard Pepping-Technical consulting. Bill Barrett-Testing coordination. Robin BroylesJohn Heise-Data acquisition. Bill Brigham-VSAR diagnostics, testing. O.B. Crump Jr.-VISAR cavity assembly. Dan Sanchez, Theresa Broyles-Testing support. Richard Wickstrom-Data reduction. Mark Heddeman-Financial support. Lloyd Bonzon-Management Support.

\section{REFERENCES}

1. L.M. Barker and RE. Hollenbach, "Shock Wove Studies of PMMA, Fused Silica, and Sapphire", Journal of Applied Physics 41:10 (Sept 1970).

2. K. J. Fleming, "Fiber Optic Coupled Sensor for Reflected Photon Analysis", Department of Energy Patent Disclosure SD-5034, S-74, 181.

3. W. F. Hemsing, "Velocity Sensing Interferometer MSAR) Modification", Review of Scientific Instruments, 50:1, (Jan 1979)

4. J. D. Matthews, L. J. Weirick, "A Hugoniot Study on PMMA Manufactured by Polycast Technology Corp.", SAND90-2402, Sandia National Laboratories, Albuquerque, NM, (Feb 1991). 